\title{
Nested sequents for the logic of conditional belief $^{\star}$
}

\author{
Marianna Girlando1 ${ }^{10000-0002-9384-1356]}$, Björn Lellmann2[0000-0002-5335-1838], \\ and Nicola Olivetti ${ }^{3}[0000-0001-6254-3754]$ \\ 1 Aix Marseille Univ, Université de Toulon, CNRS, LIS, Marseille, France and \\ University of Helsinki, Finland - marianna.girlando@univ-amu.fr \\ 2 Technische Universität Wien, Austria - lellmann@logic.at \\ 3 Aix Marseille Univ, Université de Toulon, CNRS, LIS, Marseille, France - \\ nicola.olivetti@univ-amu.fr
}

\begin{abstract}
The logic of conditional belief, called Conditional Doxastic Logic (CDL), was proposed by Board, Baltag and Smets to model revisable belief and knowledge in a multi-agent setting. We present a proof system for CDL in the form of a nested sequent calculus. To the best of our knowledge, ours is the first internal and standard calculus for this logic. We take as primitive a multi-agent version of the "comparative plausibility operator", as in Lewis' counterfactual logic. The calculus is analytic and provides a decision procedure for CDL. As a by-product we also obtain a nested sequent calculus for multi-agent modal logic $\mathrm{S} 5_{i}$.
\end{abstract}

Keywords: Nested sequent calculus · Conditional doxastic logic · Belief revision · Multi-agent epistemic logic

\section{Introduction}

Knowledge and belief are the most important propositional attitudes to reason about epistemic interaction among agents. Conditional Doxastic Logic (CDL) was proposed by Board [4] and Baltag and Smets [1-3] for modelling both belief and knowledge in a multi-agent setting (see also [14]). Differently from knowledge, the essential feature of beliefs is that they are revisable whenever the agent learns new information. To capture the revisable nature of beliefs, CDL contains the conditional belief operator $\mathrm{Bel}_{i}(C \mid B)$, the meaning of which is that agent $i$ would believe $C$ in case she learnt $B$. Both unconditional beliefs and knowledge can be defined in $\mathrm{CDL}: B e l_{i} B$ (agent $i$ believes $B$ ) as $B e l_{i}(B \mid \top), K_{i} B$ (agent $i$ knows $B)$ as $\operatorname{Bel}_{i}(\perp \mid \neg B)$, the latter meaning that $i$ considers impossible (inconsistent) to learn $\neg B$. We also consider the comparative plausibility operator $A \preccurlyeq_{i} B$, whose reading is that the agent $i$ considers $A$ to be at least as plausible as $B$. This operator, introduced by Lewis for (single-agent) counterfactual logics is interdefinable with the conditional belief operator; thereby also simple belief and knowledge can be defined directly in terms of it.

\footnotetext{
* This work was partially supported by the Project TICAMORE ANR-16-CE91-000201 and by WWTF project MA 16-28.
} 
The logic of conditional belief has been significantly employed in game theory [17], and it has been used as the basic formalism to study further dynamic extensions of epistemic logics, determined by several kinds of epistemic/doxastic actions. Not surprisingly, the axiomatization of the operator Bel in CDL internalises the well-known AGM postulates of belief revision.

The difference between the conditional belief operator $\operatorname{Bel}_{i}(B \mid A)$ and the simple belief operator $\operatorname{Bel}_{i}(A \rightarrow B)$ is illustrated by the following (modified) example from [17]. Let agent $i$ believe that Jones is a coward, $\operatorname{Bel}_{i} C(j)$. We want to express that if the agent is to learn that Jones has been sent to battle, $S(j)$, he would no longer believe that he is a coward (since only brave men are sent to battle). Using the simple belief operator would yield a contradiction, because $\neg \operatorname{Bel}_{i}(S(j) \rightarrow C(j))$ implies $\neg B e l_{i} C(j)$. However, if we express it as $\neg B e l_{i}\left(C(j) \mid S(j)\right.$ ), we retain consistency, since $\neg B e l_{i} C(j)$ cannot be derived (this is verified, e.g., using the calculus below). As a second example, consider a variant of the three-wise-men puzzle, where agent $i$ initially believes that she has a white hat, $\operatorname{Bel}_{i} W_{i}$. However, if $i$ were to learn that agent $j$ knows the colour of the hat $j$ herself wears, she would change her beliefs and be convinced that she is wearing a black hat instead: $\operatorname{Bel}_{i}\left(B_{i} \mid K_{j}\left(W_{j} \wedge B_{j}\right)\right)$. The two formulas are consistent (assuming $\left.\neg\left(B_{i} \wedge W_{i}\right)\right)$ as the operator is non-monotonic: $\operatorname{Bel}_{i}(C \mid A)$ does not entail $\operatorname{Bel}_{i}(C \mid A \wedge B)$.

The original semantics of CDL is defined in terms of Plausibility Models, i.e., standard epistemic models, where each agent is further equipped with a "comparative plausibility" relation between worlds used to evaluate her (conditional) beliefs. However, following $[8,9]$, an alternative semantics is given in terms of multi-agent neighbourhood models, which are essentially a multi-agent version of Lewis' sphere models for counterfactual logics [10]. In particular, the semantics of CDL coincides with a multi-agent version of Lewis' logic $\mathbb{V T A}$. Proof-theoretically the logic CDL has not been studied much, the only existing calculus for it being the labelled sequent calculus based on this neighbourhood semantics from [9].

Here we propose the first internal calculus for CDL, meaning that the syntactic structures employed in the calculus (nested sequents) have a direct formula translation. Since CDL admits two rather different semantics, the internal calculus presents the advantage of being independent of the choice of the semantics, differently from what happens with a labelled proof system.

Similarly to the calculi for Lewis' conditional logics in [7], our calculus $\mathcal{N}_{\mathrm{CDL}}$ takes as primitive the comparative plausibility operator, albeit in its multi-agent version $A \preccurlyeq_{i} B$. In order to obtain an internal calculus for CDL, the simple hypersequent structure used to capture Lewis' logics in [7], is no longer adequate. To keep track of the "locality" of information for each agent, and to account for beliefs of an agent occurring within the beliefs of another, we use a nested structure, which is not necessary in the single-agent case. The calculus $\mathcal{N}_{\mathrm{CDL}}$ is analytic and provides a decision procedure for CDL. Its completeness is proved semantically by extracting a finite countermodel from failed proof search. As mentioned, the epistemic operator $K_{i}$ is defined in $\mathrm{CDL}$, and it corresponds to 
the knowledge operator of multi-agent $\mathrm{S}_{i}$. Hence, "specialising" the rules of $\mathcal{N}_{\mathrm{CDL}}$ to the $K_{i}$ fragment we obtain a natural nested sequent calculus for $\mathrm{S}_{i}$.

\section{Multi-agent conditional logic CDL}

The language of CDL extends propositional logic with operators for (conditional) belief, knowledge, and comparative plausibility, all labelled with an agent.

Definition 1. Let $\mathcal{A}$ be a set of agents, and let $i$ be an agent. Formulas of CDL are generated as follows, for $P$ propositional variable:

$$
\mathcal{F}_{\mathrm{CDL}} \ni A::=P|\perp| \top|A \rightarrow A| A \preccurlyeq_{i} A \mid \operatorname{Bel}_{i}(A \mid A)
$$

A conditional belief formula $\operatorname{Bel}_{i}(C \mid B)$ is read "agent $i$ believes $C$, given $B$ ". The meaning of a formula $A \preccurlyeq_{i} B$ is that agent $i$ considers $A$ at least as plausible as $B$. The operators of $B e l_{i}$ and $\preccurlyeq_{i}$ are interdefinable:

$$
\begin{aligned}
\operatorname{Bel}_{i}(B \mid A) & \equiv\left(\perp \preccurlyeq_{i} A\right) \vee \neg((A \wedge \neg B) \preccurlyeq(A \wedge B)) \\
A \preccurlyeq_{i} B & \equiv \operatorname{Bel}_{i}(\perp \mid A \vee B) \vee \neg \operatorname{Bel}_{i}(\neg A \mid A \vee B)
\end{aligned}
$$

Intuitively, an agent conditionally believes $B$ given $A$ whenever she considers $A$ impossible or she considers $A \wedge \neg B$ to be less plausible than $A \wedge B$. Unconditional belief and knowledge can then be defined by these operators as follows ${ }^{4}$ :

$$
\begin{aligned}
& \operatorname{Bel}_{i} A:=\operatorname{Bel}_{i}(A \mid \top) \quad \operatorname{Bel}_{i} A:=\neg\left(\neg A \preccurlyeq \preccurlyeq_{i} \top\right) \quad \text { (belief) } \\
& K_{i} A:=\operatorname{Bel}_{i}(\perp \mid \neg A) \quad K_{i} A:=\perp \preccurlyeq{ }_{i} \neg A \quad \text { (knowledge) }
\end{aligned}
$$

An axiomatization of $\mathrm{CDL}$ is given by the following axioms and rules $[4,3]$ :

(0) Axiomatization of classical propositional logic

(1) If $\vdash B$, then $\vdash \operatorname{Bel}_{i}(B \mid A)$

(2) If $\vdash A \leftrightarrow B$, then $\vdash \operatorname{Bel}_{i}(C \mid A) \leftrightarrow \operatorname{Bel}_{i}(C \mid B)$

(3) $\left(\operatorname{Bel}_{i}(B \mid A) \wedge \operatorname{Bel}_{i}(B \rightarrow C \mid A)\right) \rightarrow \operatorname{Bel}_{i}(C \mid A)$

(4) $\operatorname{Bel}_{i}(A \mid A)$

(5) $\operatorname{Bel}_{i}(B \mid A) \rightarrow\left(\operatorname{Bel}_{i}(C \mid A \wedge B) \leftrightarrow \operatorname{Bel}_{i}(C \mid A)\right)$

(6) $\neg \operatorname{Bel}_{i}(\neg B \mid A) \rightarrow\left(\operatorname{Bel}_{i}(C \mid A \wedge B) \leftrightarrow \operatorname{Bel}_{i}(B \rightarrow C \mid A)\right)$

(7) $\operatorname{Bel}_{i}(B \mid A) \rightarrow \operatorname{Bel}_{i}\left(\operatorname{Bel}_{i}(B \mid A) \mid C\right)$

(8) $\neg \operatorname{Bel}_{i}(B \mid A) \rightarrow \operatorname{Bel}_{i}\left(\neg \operatorname{Bel}_{i}(B \mid A) \mid C\right)$

(9) $A \rightarrow \neg \operatorname{Bel}_{i}(\perp \mid A)$

These axioms represent an "internalised" version of the AGM belief revision postulates in a multi-agent setting, e.g., axioms 5 and 6 encode the Minimal Change Principle ${ }^{5}$ An alternative axiomatization of CDL taking $\preccurlyeq_{i}$ as primitive

\footnotetext{
${ }^{4}$ An equivalent definition of the simple belief operator is the following: $\operatorname{Bel}_{i} A:=$ $\neg\left(\neg A \preccurlyeq_{i} A\right)[10]$. We choose a simpler formulation in terms of $\top$, also from [10].

${ }^{5}$ Refer to $[4,9]$ for a detailed correspondence.
} 
essentially amounts to a multi-agent version of Lewis' counterfactual logic system $\mathbb{V T A}[7]$ and contains in addition to classical propositional logic the following:

$$
\begin{array}{ll}
(\mathrm{CPR}) \frac{\vdash B \rightarrow A}{\vdash A \preccurlyeq_{i} B} & (\mathrm{CPA})\left(A \preccurlyeq_{i} A \vee B\right) \vee\left(B \preccurlyeq_{i} A \vee B\right) \\
(\mathrm{TR})\left(A \preccurlyeq_{i} B\right) \wedge\left(B \preccurlyeq_{i} C\right) \rightarrow\left(A \preccurlyeq_{i} C\right) & (\mathrm{CO})\left(A \preccurlyeq_{i} B\right) \vee\left(B \preccurlyeq_{i} A\right) \\
(\mathrm{N}) \neg\left(\perp \preccurlyeq_{i} \mathrm{~T}\right) & (\mathrm{T})\left(\perp \preccurlyeq_{i} \neg A\right) \rightarrow A \\
\left(\mathrm{~A}_{1}\right)\left(A \preccurlyeq_{i} B\right) \rightarrow\left(\perp \preccurlyeq_{i} \neg\left(A \preccurlyeq_{i} B\right)\right) & \left(\mathrm{A}_{2}\right) \neg\left(A \preccurlyeq_{i} B\right) \rightarrow\left(\perp \preccurlyeq_{i}\left(A \preccurlyeq_{i} B\right)\right)
\end{array}
$$

The original semantics of CDL is given in terms of plausibility models; the alternative semantics in terms of neighbourhood models from [9] is as follows.

Definition 2. Let $\mathcal{A}$ be a set of agents; a multi-agent neighbourhood model has the form $\mathcal{M}=\left\langle W,\left\{N_{i}\right\}_{i \in \mathcal{A}}, \llbracket \rrbracket\right\rangle$ where $W$ is a non empty set of worlds, $\llbracket \rrbracket:$ Atm $\rightarrow \mathcal{P}(W)$ is the evaluation for atomic formulas and for each $i \in \mathcal{A}$, $N_{i}: W \rightarrow \mathcal{P}(\mathcal{P}(W))$ is a neighbourhood function, satisfying:

- Non-emptiness: For all $\alpha \in N_{i}(x), \alpha \neq \emptyset$

- Nesting: For all $\alpha, \beta \in N_{i}(x), \alpha \subseteq \beta$ or $\beta \subseteq \alpha$

- Total reflexivity: There exists $\alpha \in N_{i}(x)$ such that $x \in \alpha$

- Local absoluteness: If $\alpha \in N_{i}(x)$ and $y \in \alpha$ then $N_{i}(x)=N_{i}(y)$

The truth conditions for Boolean combinations of formulas are standard; the remaining ones use the local forcing notation introduced in [12], i.e., $\alpha \Vdash^{\forall} A$ iff for all $y \in \alpha$ we have $y \Vdash A$, and $\alpha \Vdash^{\exists} A$ iff there exists $y \in \alpha$ such that $y \Vdash A$ :

$x \Vdash \operatorname{Bel}_{i}(B \mid A)$ iff for all $\alpha \in N_{i}(x)$ it holds that $\alpha \Vdash^{\forall} \neg A$ or there exists $\beta \in N_{i}(x)$ such that $\beta \Vdash^{\exists} A$ and $\beta \Vdash^{\forall} A \rightarrow B$

$x \Vdash A \preccurlyeq_{i} B$ iff for all $\beta \in N_{i}(x)$ if $\beta \Vdash^{\exists} B$ then $\beta \Vdash^{\exists} A$

$x \Vdash B e l_{i} B$ iff there exists $\beta \in N_{i}(x)$ such that $\beta \Vdash^{\forall} B$

$x \Vdash K_{i} B$ iff for all $\beta \in N_{i}(x)$ it holds that $\beta \Vdash^{\forall} B$

A formula $A$ is valid in $\mathcal{M}$ if for all $w \in W, w \Vdash A$. A formula $A$ is valid if $A$ is valid in every multi-agent neighbourhood model.

\section{$3 \quad$ Nested sequent calculus $\mathcal{N}_{\mathrm{CDL}}$}

In this section we present a sequent for CDL. The calculus is based on the structure of nested sequents (e.g., $[5,16]$ ), adjusted to the multiagent setting and extended with the mechanism to handle comparative plausibility formulas using conditional blocks from $[6,13]$ as follows.

Definition 3. A multi-agent conditional block for agent $i$ is a syntactic structure $i:\left(A_{1} \ldots A_{n} \triangleleft_{i} B\right)$, interpreted as: $\left(A_{1} \vee \cdots \vee A_{n}\right) \preccurlyeq_{i} B . A$ multi-agent nested sequent (short: nested sequent) $S$ is a structure

$$
S=\Gamma \Rightarrow \Delta,\left[G_{1}\right]^{i_{1}}, \ldots,\left[G_{n}\right]^{i_{n}}
$$

where $i_{1}, \ldots, i_{n} \in \mathcal{A}, \Gamma$ is a multiset of formulas, and $\Delta$ is a multiset of formulas and multi-agent conditional blocks, and each $G_{1}, \ldots, G_{n}$ is a nested sequent. 
Intuitively, a nested sequent is a finite labelled directed tree with nodes labelled with sequents $\Gamma \Rightarrow \Delta$, where $\Delta$ also contains multi-agent conditional blocks, and edges labelled with agents. We call the nodes with their sequent label the components of the nested sequent. Thus each $G_{j}$ represents an immediate subtree of the tree with root $S$. The formula interpretation is given by:

$$
\begin{aligned}
& \left(\Gamma \Rightarrow \Delta,\left(\Sigma_{1} \triangleleft_{i} C_{1}\right), \ldots,\left(\Sigma_{k} \triangleleft_{j} C_{k}\right),\left[G_{1}\right]^{i_{1}}, \ldots,\left[G_{n}\right]^{i_{n}}\right)^{i n t}:= \\
& \quad \bigwedge \Gamma \rightarrow \bigvee \Delta \vee \bigvee_{1 \leqslant s \leqslant k}\left(\left(\bigvee \Sigma_{s}\right) \preccurlyeq{ }_{i} C_{s}\right) \vee K_{i_{1}}\left(G_{1}\right)^{i n t} \vee \cdots \vee K_{i_{n}}\left(G_{n}\right)^{i n t}
\end{aligned}
$$

for $K_{i} A=\perp \preccurlyeq_{i} \neg A$. We sometimes include nested successors into the succedent of a sequent, denoted with superscript *. E.g., for $\Gamma \Rightarrow \Delta,[G]^{i}$ we also write $\Gamma \Rightarrow \Delta^{*}$. For a multiset $\Delta$, we write $\operatorname{set}(\Delta)$ for its underlying set, i.e., its carrier.

To operate with nested sequents, we use the notion of context, denoting a nested sequent with a unique "hole", to be filled with another nested sequent.

Definition 4. We define a context $G\{\}$ as:

$-G\{\}=\Gamma \Rightarrow \Delta^{*},\{\}$ is a context;

- if $F\{\}$ is a context, then $G\{\}=\Gamma \Rightarrow \Delta^{*},[F\{\}]^{i}$ is a context.

The result of filling a context $G\{\}$ with a nested sequent $\Gamma \Rightarrow \Delta^{*}$ then is denoted as $G\left\{\Gamma \Rightarrow \Delta^{*}\right\}$ and defined via:

- If $G\{\}=\Sigma \Rightarrow \Pi^{*},\{\}$, then $G\left\{\Gamma \Rightarrow \Delta^{*}\right\}=\Gamma, \Sigma \Rightarrow \Delta^{*}, \Pi^{*}$;

- If $G\{\}=\Sigma \Rightarrow \Pi^{*},[F\{\}]^{i}$ then $G\left\{\Gamma \Rightarrow \Delta^{*}\right\}=\Sigma \Rightarrow \Pi^{*},\left[F\left\{\Gamma \Rightarrow \Delta^{*}\right\}\right]^{i}$.

The rules of the multi-agent nested sequent calculus $\mathcal{N}_{\mathrm{CDL}}$ are given in Fig. 1 . They are formulated in the cumulative version, repeating all formulas and blocks of the conclusion in the premisses. This is used for proving completeness, but of course could be avoided at the cost of explicit contraction rules.

As in nested calculi, each nested sequent can be thought as encoding the formulas relative to one world of the model. Since our neighbourhood models are multi-agent, each nested sequent has associated a label for an agent.

More in detail, rule $\mathrm{R} \preccurlyeq$ introduces backwards a conditional block, and rule $\mathrm{L} \preccurlyeq$ (read upwards) combines a the true plausibility formula in the antecedent of a sequent with a with the false conditional block in the consequent by means of a case analysis. With the com rule, two blocks communicate with one another. This rule can be thought as a syntactic equivalent of the nesting condition over neighbourhoods, with each conditional block encoding the comparative plausibility formulas relative to one neighbourhood of the model. The jump rule creates a new nested sequent in correspondence to a conditional block, with the same agent label. Rule $\mathrm{T}$ accounts for the condition of total reflexivity of the neighbourhood function, and the transfer rules are needed to express local absoluteness: due to this condition comparative plausibility formulas are evaluated in the same way at all the worlds accessible for the same agent - and thus, these formulas are allowed to "pass" between nested sequents with the same agent label. Finally, the rules of conditional belief make use of the definition of $\operatorname{Bel}_{i}(B \mid A)$ in terms of 
the comparative plausibility operator given in the previous section. For instance, rule Bel $\mathrm{L}_{\mathrm{L}}$ read backwards states that if $\operatorname{Bel}_{i}(B \mid A)$ is true, either $A$ is impossible (left premiss) or $A \wedge \neg B$ is strictly less plausible than $A$ (right premiss).

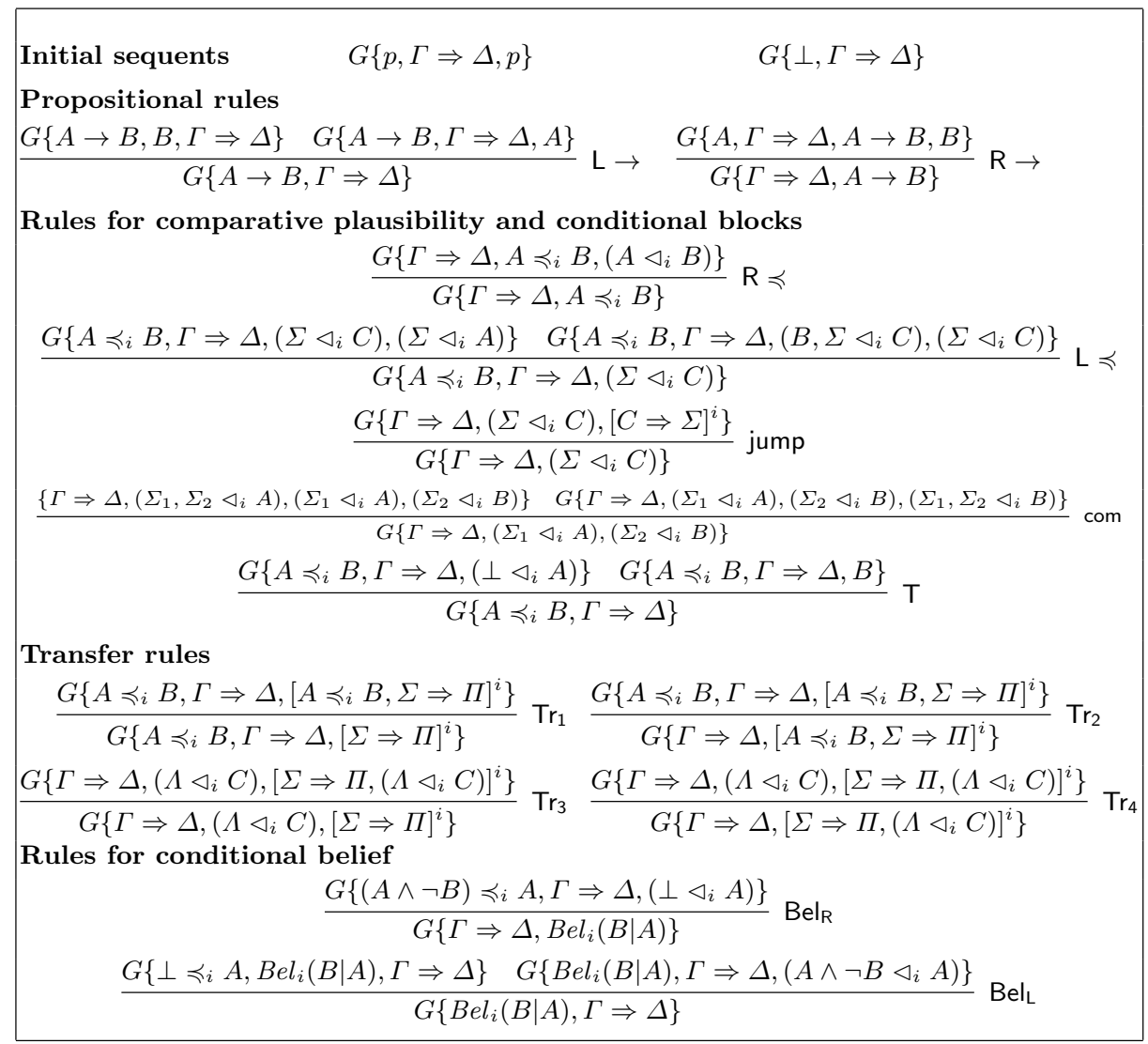

Fig. 1. Nested calculus $\mathcal{N}_{\mathrm{CDL}}$

Theorem 1 (Soundness). If $G$ is derivable in $\mathcal{N}_{\mathrm{CDL}}$ then $(G)^{\text {int }}$ is valid.

Proof. By induction on the derivation height, showing that if the premiss of a rule is valid, so is its conclusion. By means of example we show jump, $\mathrm{T}$ and $\mathrm{Tr}_{1}$.

Suppose the premiss of jump is valid, and its conclusion is not. Thus, there exists a model such that $\mathcal{M}, x \Vdash F$ for all $F \in \Gamma$ and $\mathcal{M}, x \nVdash H$ for all $H \in \Delta$. Since $x \nVdash(\Sigma \triangleleft C)$, there exists $\alpha \in N_{i}(x)$ such that $\alpha \Vdash^{\exists} C$ and $\alpha \nVdash^{\exists}$ $\left(A_{1} \vee \cdots \vee A_{n}\right)$, for $\Sigma=A_{1}, \ldots, A_{n}$. Then there exists $y \in \alpha$ such that $\mathcal{M}, y \Vdash C$ and $\mathcal{M}, y \nVdash\left(A_{1} \vee \cdots \vee A_{n}\right)$. However, from the previous conditions and validity of the premiss we have that for all $k \in \bigcup N_{i}(x)$ either $\mathcal{M}, k \nVdash C$ or $\mathcal{M}, k \Vdash A_{s}$, for some $A_{s} \in \Sigma$, contradicting the latter statement. 
As for $\mathrm{T}$, suppose the premisses of the rule are valid, while the conclusion is not. Thus, there is a model $\mathcal{M}, x \Vdash A \preccurlyeq_{i} B$, such that for all $F \in \Gamma, H \in \Delta$, $\mathcal{M}, x \Vdash F$ and $\mathcal{M}, x \nVdash H$. From $\mathcal{M}, x \Vdash A \preccurlyeq{ }_{i} B$ we have for all $\alpha \in N_{i}(x)$, if $\alpha \Vdash^{\exists} B$, then $\alpha \Vdash^{\exists} A$. As for the premisses, it must hold that $\mathcal{M}, x \Vdash \perp \preccurlyeq i$, and thus that $(*)$ for all $\alpha \in N_{i}(x), \alpha \|^{\exists} A$ and $\mathcal{M}, x \Vdash B$. By total reflexivity, there is a $\beta \in N_{i}(x)$ such that $x \in \beta$. Thus, $\beta \Vdash^{\exists} B$, whence $\beta \Vdash^{\exists} A$, which contradicts $(*)$.

Similarly, suppose the premiss of $\operatorname{Tr}_{1}$ is valid, while the conclusion is not. Then there is a model such that $\mathcal{M}, x \Vdash A \preccurlyeq_{i} B$, and for all $F \in \Gamma, H \in \Delta$, $\mathcal{M}, x \Vdash F$ and $\mathcal{M}, x \nVdash H$. Moreover, we have that there exists $y \in \bigcup N_{i}(x)$ such that $\mathcal{M}, y \Vdash S$ for all $S \in \Sigma$, and $\mathcal{M}, y \nVdash P$, for all $P \in \Pi$. From all these conditions, and from the fact that the premiss of $\operatorname{Tr}_{1}$ are assumed to be valid, we obtain in particular that $(\star) \mathcal{M}, y \nVdash A \preccurlyeq{ }_{i} B$. However, by local absoluteness we have $N_{i}(x)=N_{i}(y)$; thus $\mathcal{M}, x \Vdash A \preccurlyeq{ }_{i} B$, against $(\star)$.

Lemma 1. The rules of weakening and contraction are admissible in $\mathcal{N}_{\mathrm{CDL}}$ :

$$
\frac{G\left\{\Gamma \Rightarrow \Delta^{*}\right\}}{G\left\{\Gamma, \Sigma \Rightarrow \Delta^{*}, \Pi^{*}\right\}} \mathrm{W} \quad \frac{G\left\{\Gamma, A, A \Rightarrow \Delta^{*}\right\}}{G\left\{\Gamma, A \Rightarrow \Delta^{*}\right\}} \mathrm{C}_{L} \quad \frac{G\left\{\Gamma \Rightarrow \Delta^{*}, A, A\right\}}{G\left\{\Gamma \Rightarrow \Delta^{*}, A\right\}} \mathrm{C}_{R}
$$

Proof. Standard, by induction on the depth of the derivation.

Remark 1. The rules for simple belief and knowledge can be explicitly defined as follows:

$$
\begin{gathered}
\frac{G\left\{\Gamma \Rightarrow \Delta,\left(\neg A \triangleleft_{i} \top\right)\right\}}{G\left\{\operatorname{Bel}_{i} A, \Gamma \Rightarrow \Delta\right\}} \mathrm{B}_{\mathrm{L}} \quad \frac{G\{\neg A \preccurlyeq i \top, \Gamma \Rightarrow \Delta\}}{G\left\{\Gamma \Rightarrow \Delta, B e l_{i} A\right\}} \mathrm{B}_{\mathrm{R}} \\
\frac{G\left\{K_{i} A, \Gamma \Rightarrow \Delta,\left(\neg A, \Sigma \triangleleft_{i} C\right)\right\}}{G\left\{K_{i} A, \Gamma \Rightarrow \Delta,\left(\Sigma \triangleleft_{i} C\right)\right\}} \mathrm{K}_{\mathrm{L}} \frac{G\left\{\Gamma \Rightarrow \Delta,\left(\perp \triangleleft_{i} A\right)\right\}}{G\left\{\Gamma \Rightarrow \Delta, K_{i} A\right\}} \mathrm{K}_{\mathrm{R}}
\end{gathered}
$$

Example 1. A derivation of $K_{i} A \rightarrow \operatorname{Bel}_{i}\left(\neg \operatorname{Bel}_{j}(\perp \mid A)\right)$ is shown in Fig. 2, with rule $\mathrm{R} \neg$ derivable from $\mathrm{R} \rightarrow$, recalling $\neg A=A \rightarrow \perp$. We omit repetitions of the principal formulas in the premisses.

\section{Completeness of $\mathcal{N}_{\mathrm{CDL}}$}

To prove completeness of $\mathcal{N}_{\mathrm{CDL}}$, we show how to construct a countermodel from failed proof-search. For this, we first introduce the notion of saturated sequent (Definition 7), i.e., an unprovable sequent to which all the rules have been nonredundantly applied. Then, we build a countermodel for the sequent placed at the root of the derivation from the information contained in the saturated sequent.

Intuitively, we can consider a saturated sequent $S$ as a labelled tree, where each node is a nested component $S_{j}$ of $S$. Each world of a countermodel for $S$ corresponds to a node of the tree, and the world falsifying $S$ as a whole is the node placed at the root of the tree.

In countermodel construction we have to take care of the following: (a) for each agent $i$ and world $S_{j}$ define a system of neighbourhoods $N_{i}\left(S_{j}\right)$; and (b) 


$$
\begin{aligned}
& \frac{\operatorname{Bel}_{j}(\perp \mid A) \preccurlyeq_{i} K_{i} A \Rightarrow\left[\perp \preccurlyeq_{i} A, \operatorname{Bel}_{j}(\perp \mid A), A \Rightarrow \perp, A\right]^{i}}{\operatorname{Bel}_{j}(\perp \mid A) \preccurlyeq_{i} K_{i} A \Rightarrow\left[\perp \preccurlyeq_{i} A, \operatorname{Bel}_{j}(\perp \mid A) \Rightarrow \neg A, \perp, A\right]^{i}} \mathrm{R}- \\
& \operatorname{Bel}_{j}(\perp \mid A) \preccurlyeq_{i} K_{i} A \Rightarrow\left[\perp \preccurlyeq_{i} A, \operatorname{Bel}_{j}(\perp \mid A) \Rightarrow \neg A, \perp\right]^{i}
\end{aligned}
$$

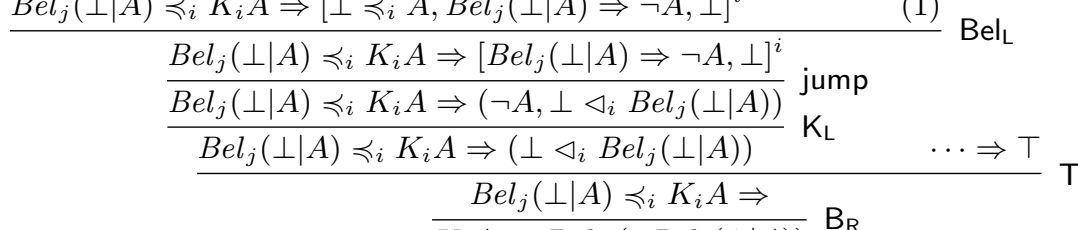

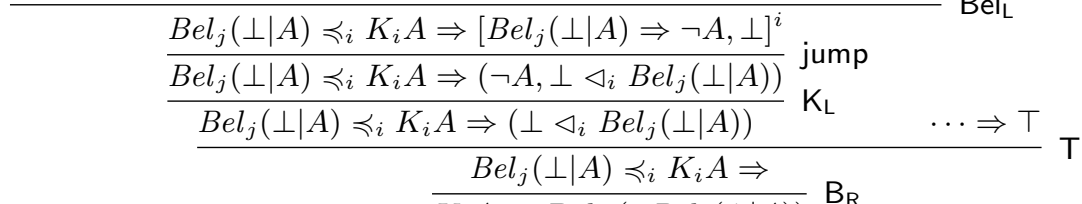

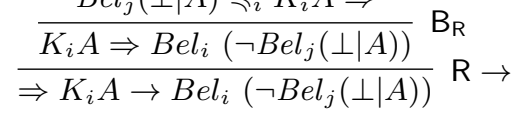

The derivations of sequents (1) and (2) respectively are:

$$
\begin{gathered}
\frac{\cdots \Rightarrow\left[\cdots \Rightarrow \neg A, \perp,[A \Rightarrow A]^{j}\right]^{i} \quad \cdots \Rightarrow\left[\cdots \Rightarrow \neg A, \perp,[A \Rightarrow \top]^{j}\right]^{i}}{B e l_{j}(\perp \mid A) \preccurlyeq_{i} K_{i} A \Rightarrow\left[B e l_{j}(\perp \mid A) \Rightarrow \neg A, \perp,[A \Rightarrow A \wedge \top]^{j}\right]^{i}} \text { R^ } \\
\operatorname{Bel}_{j}(\perp \mid A) \preccurlyeq_{i} K_{i} A \Rightarrow\left[B e l_{j}(\perp \mid A) \Rightarrow \neg A, \perp,\left(A \wedge \top \triangleleft_{j} A\right)\right]^{i} \\
\frac{B e l_{j}(\perp \mid A) \preccurlyeq_{i} K_{i} A \Rightarrow\left[B e l_{j}(\perp \mid A) \Rightarrow \neg A, \perp,[\perp \Rightarrow \perp]^{j}\right]^{i}}{B e l_{j}(\perp \mid A) \preccurlyeq_{i} K_{i} A \Rightarrow\left[B e l_{j}(\perp \mid A) \Rightarrow \neg A, \perp,\left(\perp \triangleleft_{j} \perp\right)\right]^{i}} \text { jump }
\end{gathered}
$$

Fig. 2. Derivation of the formula $K_{i} A \rightarrow \operatorname{Bel}_{i}\left(\neg B e l_{j}(\perp \mid A)\right)$.

verify that the condition of local absoluteness holds in the model. Concerning (a), the neighbourhoods $N_{i}\left(S_{j}\right)$ will be determined by the blocks $\left(\Sigma \triangleleft_{i} C\right)$ contained in the consequent of $S_{j}$. As for (b), we need our models to satisfy the following property. Let $\mathcal{M}$ be an arbitrary model, $x, y$ two worlds in the model, and $R_{i}(x, y)$ the relation defined as $y \in \bigcup N_{i}(x)$. By local absoluteness it follows that $R_{i}$ is an equivalence relation ${ }^{6}$ and from $R_{i}(x, y)$ follows $N_{i}(y)=$ $N_{i}(x)$. The syntactic counterpart of $R_{i}$ is the equivalence relation $\sim_{i}$ between two components $S_{j}$ and $S_{k}$ of $S$, one of which might be $S$ itself (Definition 6 ). This relation holds whenever $S_{j}$ and $S_{k}$ are related by an $i$-path in the tree associated with $S$. Lemma 2 proves that if $S_{j} \sim_{i} S_{k}$ then the two nested sequents contain the same blocks. This suffices to ensure that $N_{i}\left(S_{j}\right)=N_{i}\left(S_{k}\right)$.

Let us come back to (a). To define the set $N_{i}\left(S_{j}\right)$ for a world $S_{j}$, we consider the blocks $\left(\Sigma \triangleleft_{i} C\right)$ occurring in the consequent of $S_{j}$. However since the rules are cumulative, $S_{j}$ may contain two blocks $\left(A_{1}, A_{2} \triangleleft_{i} C\right)$ and $\left(A_{1}, A_{2}, A_{3} \triangleleft_{i}\right.$ $C)$. In this case the former block can be disregarded, as it is included in the latter. Thus, only "maximal" blocks (Definition 8) are relevant in order to define $N_{i}\left(S_{j}\right)$. It turns out that maximal blocks of a saturated sequent are ordered by set inclusion, due to the com rule. Moreover, each maximal block ( $\Sigma \triangleleft_{i}$ $C$ ) occurring in $S_{j}$ is supposed to be false in world $S_{j}$. This means that $S_{j}$ has associated a "witnessing" world $S_{k}$ where $C$ is true and all formulas in

\footnotetext{
${ }^{6}$ Refer to next section on $\mathrm{S}_{i}$.
} 
$\Sigma$ are false. This world / component is such that $S_{j} \sim_{i} S_{k}$, and its existence is guaranteed by saturation with respect to jump. Thus, the neighbourhoods $N_{i}\left(S_{j}\right)$ are determined by the maximal blocks and their witnessing worlds. The following example should illustrate the construction.

Example 2. For $p_{i}, r, s, t, u$, distinct atomic formulas, let:

$$
\begin{gathered}
\Pi=\left(p_{1} \triangleleft_{i} r\right),\left(p_{1} \triangleleft_{i} s\right),\left(p_{1}, p_{2} \triangleleft_{i} t\right),\left(p_{1}, p_{2}, p_{3} \triangleleft_{i} u\right) \\
S=c \Rightarrow \Pi,\left[r \Rightarrow p_{1}, \Pi\right]^{i},\left[s \Rightarrow p_{1}, \Pi\right]^{i},\left[t \Rightarrow p_{1}, p_{2}, \Pi\right]^{i},\left[u \Rightarrow p_{1}, p_{2}, p_{3}, \Pi\right]^{i}
\end{gathered}
$$

The four components of $S$ are numbered as $S_{1}, S_{2}, S_{3}, S_{4}$ respectively (so that $S_{1}=\left[r \Rightarrow p_{1}, \Pi\right]^{i}$ etc.). Sequent $S$ is saturated according to Definition 7 . Moreover, observe that the blocks in $\Pi$ are ordered by set inclusion, and that each block has an associated witnessing world: $\left(p_{1} \triangleleft_{i} r\right)$ is associated to $S_{1},\left(p_{1} \triangleleft_{i} s\right)$ to $S_{2},\left(p_{1}, p_{2} \triangleleft_{i} t\right)$ to $S_{3}$ and $\left(p_{1}, p_{2}, p_{3} \triangleleft_{i} u\right)$ to $S_{4}$. In the countermodel, $W=\left\{S, S_{1}, S_{2}, S_{3}, S_{4}\right\}$. The system of neighbourhoods $N_{i}(S)$ is determined by putting in the smallest neighbourhood the worlds corresponding to the largest block, and so on.

$$
N_{i}(S)=\left\{\left\{S_{4}\right\},\left\{S_{4}, S_{3}\right\},\left\{S_{4}, S_{3}, S_{2}, S_{1}\right\},\left\{S_{4}, S_{3}, S_{2}, S_{1}, S\right\}\right\}
$$

This ensures that if a neighbourhood $\alpha$ falsifies a block $\left(\Sigma \triangleleft_{i} C\right)$, i.e., $\alpha \Vdash^{\exists} C$ and $\alpha \forall^{\exists} \bigvee \Sigma$, then any larger neighbourhood falsifies the block as well. The inclusion of $S$ in the largest sphere is needed to ensure total reflexivity. Since the worlds are related by $\sim_{i}$, we have that $N_{i}\left(S_{j}\right)=N_{i}(S)$. Finally, the evaluation function assigns to atoms the worlds / nested component containing the atoms in the antecedent. Thus, $\llbracket u \rrbracket=\left\{S_{4}\right\}, \llbracket t \rrbracket=\left\{S_{3}\right\}, \llbracket u \rrbracket=\left\{S_{2}\right\}, \llbracket r \rrbracket=\left\{S_{1}\right\}, \llbracket c \rrbracket=\{S\}$. It can be easily seen that world $S$ falsifies sequent $S$ : for instance, in case of block $\left(p_{1}, p_{2} \triangleleft_{i} t\right)$, we have $\{S 4, S 3\} \Vdash^{\exists} t$ but $\{S 4, S 3\} \forall^{\exists} p_{1} \vee p_{2}$.

Definition 5. Let $S_{1}$ and $S_{2}$ be two nested sequents. We say that $S_{2}$ occurs in $S_{1}$, in symbols $S_{2} \tilde{\in} S_{1}$ if $S_{1}=S_{2}$ or $S_{1}=\Gamma \Rightarrow \Delta^{*},\left[S_{3}\right]^{i}$ for some $i$ and $S_{2} \tilde{\in} S_{3}$.

Viewing nested sequents as labelled trees, we thus have $S_{2} \tilde{\in} S_{1}$ if $S_{2}$ is a subtree of $S_{1}$. We denote by the symbol $\in$ occurrence of a formula $A$ or conditional block $\left(\Sigma \triangleleft_{i} A\right)$ in a multiset $\Gamma$ of formulas and conditional blocks.

Definition 6. Let $S$ be a nested sequent. For every agent $i$ the relation $\sim_{i}$ on the nested sequents occurring in $S$ is the equivalence relation generated by the relation $\sim_{i}^{1}$ given by: $S_{1} \sim_{i}^{1} S_{2}$ iff $S_{1}=\Gamma \Rightarrow \Delta,\left[S_{2}\right]^{i}$.

Intuitively, we have $S_{1} \sim_{i} S_{2}$ if $S_{1}=S_{2}$ or the two components are linked with an $i$-path. Next, recall that $\operatorname{set}(\Delta)$ is the set underlying the multiset $\Delta$.

Definition 7. Let $S=\Gamma \Rightarrow \Delta,\left[G_{1}\right]^{i_{1}}, \ldots\left[G_{n}\right]^{i_{n}}$ be a nested sequent. We say that $S$ is locally saturated if it satisfies the following conditions.

1. (init) $\Gamma \cap \Delta=\emptyset$ and $\perp \notin \Gamma$; 
2. ( $\mathrm{L} \rightarrow$ ) If $A \rightarrow B \in \Gamma$ then $A \in \Delta$ or $B \in \Gamma$;

3. ( $\mathrm{R} \rightarrow$ ) If $A \rightarrow B \in \Delta$ then $A \in \Gamma$ and $B \in \Delta$;

4. $(\mathrm{R} \preccurlyeq)$ If $A \preccurlyeq_{i} B \in \Delta$ then there exists a conditional block $\left(A \triangleleft_{i} B\right) \in \Delta$;

5. $(\mathrm{L} \preccurlyeq)$ If $A \preccurlyeq \preccurlyeq_{i} B \in \Gamma$ and $\left(\Sigma \triangleleft_{i} C\right) \in \Delta$, then there is $a\left(\Sigma^{\prime} \triangleleft_{i} C\right) \in \Delta$ with $\operatorname{set}(\Sigma, B)=\operatorname{set}\left(\Sigma^{\prime}\right)$ or $\left(\Sigma \triangleleft_{i} A\right) \in \Delta$;

6. (com) If $\left(\Sigma_{1} \triangleleft_{i} A\right)$ and $\left(\Sigma_{2} \triangleleft_{i} B\right) \in \Delta$, then for some $\Pi$ with $\operatorname{set}\left(\Sigma_{1}, \Sigma_{2}\right) \subseteq$ $\operatorname{set}(\Pi)$ we have $\left(\Pi \triangleleft_{i} A\right) \in \Delta$ or $\left(\Pi \triangleleft_{i} B\right) \in \Delta$.

7. (T) If $A \preccurlyeq_{i} B \in \Delta$ then either $\left(\perp \triangleleft_{i} A\right) \in \Delta$ or $B \in \Delta$;

We denote by $\operatorname{Block}_{i}(S)$ the set of conditional blocks in $\Delta$ labelled with $i$. Moreover, we say that $S$ is saturated if the following conditions hold for every $S_{1} \tilde{\in} S$ :

$-S_{1}$ is locally saturated;

- (jump) If $S_{1}=\Gamma \Rightarrow \Delta^{*},\left(\Sigma \triangleleft_{i} C\right)$, then there is a $S_{2} \sim_{i} S_{1}$ with $S_{2}=\Phi \Rightarrow$ $\Omega^{*},\left[\Psi, C \Rightarrow \Sigma, \Xi^{*}\right]^{i}$

- (Transfer rules) If $S_{1}=\Gamma \Rightarrow \Delta^{*},\left[\Sigma \Rightarrow \Pi^{*}\right]^{i}$, then $\operatorname{Block}_{i}\left(S_{1}\right)=\operatorname{Block}_{i}(\Sigma \Rightarrow$ $\left.\Pi^{*}\right)$ and for every formula $A \preccurlyeq_{i} B$ we have $A \preccurlyeq_{i} B \in \Gamma$ iff $A \preccurlyeq_{i} B \in \Sigma$;

Lemma 2. If $S_{1}$ and $S_{2}$ are saturated and $S_{1} \sim_{i} S_{2}$, then $\operatorname{Block}_{i}\left(S_{1}\right)=\operatorname{Block}_{i}\left(S_{2}\right)$.

Proof. By induction on the length of the $i$-path between $S_{1}$ and $S_{2}$, using the saturation condition for the transfer rules in the base case.

We define a naive backwards proof-search strategy for $\mathcal{N}_{\mathrm{CDL}}$ as follows: Apply the rules bottom-up to the nested sequent unless the saturation condition associated to the particular application of the rule is already satisfied. If the sequent is saturated and not an initial sequent, return it, otherwise return "derivable".

Lemma 3. Let $S$ be a nested sequent. Then proof search under the strategy above terminates and yields a derivation or a saturated nested sequent.

Proof. For termination, we first bound the number of the nested sequents occurring in the proof search. Let $n$ be the size of $S$, i.e., the number of symbols occurring in it. Note that the premisses of the rules contain at least one formula occurrence more than the conclusion. Since according to the proof-search strategy rules are not applied if the nested sequent already satisfies the corresponding saturation condition, no formula or block is added twice. Since $S$ contains at most $n$ many formulas, at most $2^{n} \cdot n$ many different conditional blocks and $2^{n} \cdot 2^{n}$ many sequents consisting only of formulas can be obtained without repetition. Hence at most $2^{2^{n} \cdot n} \cdot 2^{2 n}$ many different sequents consisting of formulas and blocks occur in the proof search. To bound the maximal depth of a nested sequent (seen as a tree) occurring in the proof search, we consider a branch in such a nested sequent and divide it into blocks, taking two components $S_{1}$ and $S_{2}$ in the branch to be in the same block if for some agent $i$ we have $S_{1} \sim_{i} S_{2}$. Since the maximal nesting depth of comparative plausibility formulas in $S$ is $n$, the number of alternations between agents in such a formula is at most $n$. Every application of the jump rule produces a new component such that the maximal nesting depth of formulas in this component is strictly smaller than 
that of the component from which it was created. Moreover the transfer rules only transfer comparative plausibility formulas and blocks across nesting operators for the same agent. Hence every branch of every nested sequent occurring in the proof search contains at most $n$ many non-trivial blocks in addition to those of $S$. Thus the maximal depth of a nested sequent occurring in the proof search is the number of possible sequents times the maximal number of blocks in a branch, i.e., $2^{2^{n} \cdot n} \cdot 2^{2 n} \cdot 2 n=\mathcal{O}\left(2^{2^{n}}\right)$. Since the branching of the nested sequents themselves (seen as trees) is caused by applications of the jump rule, by the saturation conditions the branching of a nested sequent is bounded by the number of formula-formula sequents, i.e., $2^{2 n}$. Hence the number of components of a nested sequent occurring in the proof search is $\mathcal{O}\left(\left(2^{2^{n}}\right)^{2^{2 n}}\right)$. Further, each of these components contains one of at most $\mathcal{O}\left(2^{2^{n}}\right)$ many sequents. Hence the total number of nested sequents which might occur in the proof search is finite. Together with the fact that in every step of the proof search at least one new occurrence of a formula is added, this means that the algorithm terminates.

It is straightforward to construct a derivation if the procedure returns "derivable". Suppose that it does not yield a derivation. Since the algorithm terminates, it yields a nested sequent $S$. But this nested sequent must satisfy the saturation conditions for every rule, since otherwise it would be possible to apply the corresponding rule and the procedure would not have terminated.

We then construct a countermodel from a saturated nested sequent. While the worlds of the model will be the components of the nested sequents, for defining the neighbourhood function we consider the "largest" blocks in the components:

Definition 8. For a nested sequent $S$, a conditional block $\left(\Sigma \triangleleft_{i} C\right) \in \operatorname{Block}_{i}(S)$ is maximal if there is no block $\left(\Sigma^{\prime} \triangleleft_{i} C\right) \in \operatorname{Block}_{i}(S)$ with $\operatorname{set}(\Sigma) \subsetneq \operatorname{set}\left(\Sigma^{\prime}\right)$. We write $\operatorname{MaxBlock}_{i}(S)$ for the set of maximal blocks in $\operatorname{Block}_{i}(S)$.

Remark 2. A maximal conditional block is the "largest" (containing most formulas in the antecedent) of all the blocks in $\operatorname{Block}_{i}(S)$ with the same consequent. Thus, all maximal blocks have a different consequent. If $S$ is saturated, the antecedents of the conditional blocks in $\operatorname{MaxBlock}_{i}(S)$ can be ordered w.r.t. set inclusion, such that $\operatorname{set}\left(\Sigma_{1}\right) \subset \operatorname{set}\left(\Sigma_{2}\right) \subset \cdots \subset \operatorname{set}\left(\Sigma_{k}\right)$, for $k$ the number of maximal conditional blocks. Note that there could be maximal blocks sharing the same antecedent, e.g., as a consequence of saturation with respect to $\mathrm{Tr}_{3}$, $\mathrm{Tr}_{4}$ or com, this latter applied to two different pairs of conditional blocks.

Given a saturated nested sequent $S$ as above, the construction of the countermodel $\mathcal{M}_{\mathcal{N}}=\left\langle W,\left\{N_{i}\right\}_{i \in \mathcal{A}}, \llbracket \rrbracket\right\rangle$ proceeds as follows.

$-W:=\left\{S_{j} \mid S_{j} \tilde{\in} S\right\}$

$-\llbracket p \rrbracket:=\left\{S_{j} \in W \mid p \in \Phi_{j}\right\}$.

To define the neighbourhood functions, observe that by the condition of absoluteness, this must be the same for all worlds seen by the same agent. Thus, for all nested sequents $S_{m}$ with $S_{m} \sim_{i} S_{j}$, we define a single neighbourhood function $N_{i}\left(S_{j}\right)=N_{i}\left(S_{m}\right)$. In order to do so, we consider the maximal blocks occurring 
in $S_{j}$, knowing by Lemma 2 that if $S_{j} \sim_{i} S_{m}$ then $\operatorname{Block}_{i}\left(S_{j}\right)=\operatorname{Block}_{i}\left(S_{m}\right)$, and hence $\operatorname{MaxBlock}_{i}\left(S_{j}\right)=\operatorname{MaxBlock}_{i}\left(S_{m}\right)$. Suppose the set $\operatorname{MaxBlock}_{i}\left(S_{j}\right)$ contains $n_{1}+n_{2}+\cdots+n_{k}$ maximal conditional blocks, with exactly $k$ different sets $\operatorname{set}\left(\Sigma_{1}\right) \subset \operatorname{set}\left(\Sigma_{2}\right) \subset \cdots \subset \operatorname{set}\left(\Sigma_{k}\right)$ :

$$
\begin{array}{ccc}
\left(\Sigma_{1} \triangleleft_{i} C_{1}^{1}\right) & \ldots, & \left(\Sigma_{1} \triangleleft_{i} C_{n_{1}}^{1}\right) \\
\left(\Sigma_{2} \triangleleft_{i} C_{1}^{2}\right) & \ldots, & \left(\Sigma_{2} \triangleleft_{i} C_{n_{2}}^{2}\right) \\
\vdots & \vdots \\
\left(\Sigma_{k} \triangleleft_{i} C_{1}^{k}\right) & \ldots, & \left(\Sigma_{k} \triangleleft_{i} C_{n_{k}}^{k}\right)
\end{array}
$$

So for each $z \leqslant k$ there are $n_{z}$ different blocks $\left(\Sigma_{z} \triangleleft_{i} C_{1}^{z}\right), \ldots,\left(\Sigma_{z} \triangleleft_{i} C_{n_{z}}^{z}\right)$ with the same antecedent. By the saturation condition for jump, for all $\Sigma_{z}, C_{w}^{z}$ with $w \in\left\{1, \ldots, n_{z}\right\}$, there is a $S_{z, w}=\Phi_{z, w} \Rightarrow \Omega_{z, w} \tilde{\in} S$ with $S_{j} \sim_{i} S_{z, w}, C_{w}^{z} \in \Phi_{z, w}$ and $\Sigma_{z} \subseteq \Omega_{z, w}$. Let $W_{i}^{S_{j}}=\left\{S_{z} \mid S_{z} \sim_{i} S_{j}\right\}$. Now define $N_{i}\left(S_{j}\right)$ as follows:

$$
\begin{aligned}
N_{i}\left(S_{j}\right):=\left\{\left\{S_{k, 1}, \ldots, S_{k, n_{k}}\right\},\left\{S_{k, 1}, \ldots, S_{k, n_{k}}, S_{k-1,1}, \ldots, S_{k-1, n_{k-1}}\right\}, \ldots,\right. \\
\\
\left.\left\{S_{k, 1}, \ldots, S_{k, n_{k}}, S_{k-1,1}, \ldots, S_{k-1, n_{k-1}}, \ldots S_{1,1}, \ldots, S_{1, n_{1}}\right\}, W_{i}^{S_{j}}\right\}
\end{aligned}
$$

I.e., we add into the same neighbourhood the worlds associated to blocks sharing the same antecedent. The so defined $\mathcal{M}_{\mathcal{N}}$ is a model for CDL: it satisfies the properties of non-emptiness, nesting and local absoluteness (immediate from the definition). Total reflexivity follows from the fact that for all $S_{j}, W_{i}^{S_{j}} \in N_{i}\left(S_{j}\right)$.

Lemma 4. Let $S$ be a saturated nested sequent and $S_{j}=\Phi_{j} \Rightarrow \Omega_{j}^{*}$ a nested sequent with $S_{j} \sim_{i} S$. Let $\mathcal{M}_{N}$ be the model as just defined. Let $\operatorname{MaxBlock}_{i}\left(S_{j}\right)=$ $\left(\Sigma_{1} \triangleleft_{i} C_{1}^{1}\right), \ldots,\left(\Sigma_{1} \triangleleft_{i} C_{n_{1}}^{1}\right), \ldots,\left(\Sigma_{k} \triangleleft_{i} C_{1}^{k}\right), \ldots,\left(\Sigma_{k} \triangleleft_{i} C_{n_{k}}^{k}\right)$. For $A$ a formula and $\left(\Sigma \triangleleft_{i} C\right)$ a conditional block the following hold:

1. If $A \in \Phi_{j}$ then $\mathcal{M}_{N}, S_{j} \Vdash A$;

2. If $A \in \Omega_{j}^{*}$ then $\mathcal{M}_{N}, S_{j} \nVdash A$;

3. If $\left(\Sigma \triangleleft_{i} C\right) \in \Omega_{j}^{*}$ then $\mathcal{M}_{N}, S_{j} \nVdash\left(\bigvee_{B \in \Sigma} B \preccurlyeq{ }_{i} C\right)$.

Proof. We prove statements 1 and 2 by induction on the complexity of $A$, showing only the case of comparative plausibility formulas. The proof of statement 3 uses the proof of 2 . As for 1 , suppose $A \preccurlyeq_{i} B \in \Phi_{j}$. We have to show that $\mathcal{M}_{N}, S_{j} \Vdash A \preccurlyeq_{i} B$, i.e. that for all the $\alpha \in N_{i}\left(S_{j}\right)$ we have $\alpha \nVdash^{\exists} B$ or $\alpha \Vdash^{\exists} A$. First, suppose $\alpha \neq W_{i}^{S_{j}}$. Then, $\alpha=\left\{S_{k, 1}, \ldots, S_{k, n_{k}}, \ldots, S_{t, 1}, \ldots, S_{t, n_{t}}\right\}$, for some $t \leqslant k$. For $z \leqslant k$ and $w \in\left\{1, \ldots, n_{z}\right\}$, each $S_{z, w}$ comes from a maximal conditional block $\left(\Sigma_{z} \triangleleft_{i} C_{w}^{z}\right)$, and denotes a nested sequent $\Phi_{z, w} \Rightarrow \Omega_{z, w}$ occurring in $W$ with $C_{w}^{z} \in \Phi_{z, w}$ and $\Sigma_{z} \subseteq \Omega_{z, w}$. By saturation condition $\mathrm{L} \preccurlyeq$, either $B \in \Sigma_{t}$ or $A=C_{q}^{t}$, for some $q \in\left\{t, \ldots, n_{t}\right\}$. In the former case, by $\operatorname{set}\left(\Sigma_{t}\right) \subset \operatorname{set}\left(\Sigma_{t+1}\right) \subset \cdots \subset \operatorname{set}\left(\Sigma_{k}\right)$ and by inductive hypothesis, we have that for all $S_{z, w}$, with $z \leqslant k$ and $w \in\left\{1, \ldots, n_{z}\right\}, \mathcal{M}_{N}, S_{z, w} \nVdash B$; thus, $\alpha \nVdash^{\exists} B$. Otherwise, let $A=C_{q}^{t}$, for some $q \in\left\{1, \ldots, n_{t}\right\}$. Then, $S_{t, q}=A, \Phi_{t, q}^{\prime} \Rightarrow \Omega_{t, q}$. By inductive hypothesis and since $S_{t, q} \in \alpha$ we get $\alpha \Vdash^{\exists} A$.

If $\alpha=W_{i}^{S_{j}}$, we have to prove that $W_{i}^{S_{j}} \nVdash^{\exists} B$ or $W_{i}^{S_{j}} \Vdash^{\exists} A$. Let $W_{i}^{S_{j}}=$ $\left\{S_{1}, \ldots, S_{t}\right\}$. By the saturation conditions for $\operatorname{Tr}_{1}$ and $\operatorname{Tr}_{2}$ we have $A \preccurlyeq{ }_{i} B \in \Phi_{q}$, 
for all $q \leqslant t$. By saturation condition $\mathrm{T}$, either there exists some $S_{q}$ with $\left(\perp \triangleleft_{i}\right.$ $A) \in \operatorname{Block}_{i}\left(S_{q}\right)$, or for all $S_{q}$ we have $B \in \Omega_{q}$. In the former case, by saturation condition jump, to $S_{q}$ is associated a nested sequent $S_{q^{\prime}}=A, \Phi_{q^{\prime}} \Rightarrow \Omega_{q^{\prime}}$. It holds that $S_{q} \sim_{i}^{1} S_{q^{\prime}}$, and thus $S_{q^{\prime}} \in W_{i}^{S_{j}}$. By inductive hypothesis, $\mathcal{M}, S_{q^{\prime}} \Vdash A$, and $W_{i}^{S_{j}} \Vdash^{\exists} A$. Otherwise, we have that for all $S_{q}, B \in \Omega_{q}$. By inductive hypothesis $\mathcal{M}, S_{q} \nVdash B$, and thus $W_{i}^{S_{j}} \nVdash^{\exists} B$.

As for 2 , suppose $A \preccurlyeq_{i} B \in \Omega_{j}$. We have to prove that $\mathcal{M}_{N}, S_{j} \nVdash A \preccurlyeq B$, i.e., that there is an $\alpha \in N_{i}\left(S_{j}\right)$ with $\alpha \Vdash^{\exists} B$ and $\alpha \Vdash^{\exists} B$ and $\alpha \nVdash^{\exists} A$. From the definition of $N_{i}\left(S_{j}\right)$, and with $z \leqslant k$ and $w \in\left\{1, \ldots, n_{z}\right\}$, we have that to each $S_{z, w}$ occurring in $\bigcup N_{i}\left(S_{j}\right)$ is associated a sequent $C_{w}^{z}, \Phi_{z, w} \Rightarrow \Omega_{z, w}, \Sigma_{z}$, coming from a maximal conditional block $\left(\Sigma_{z} \triangleleft_{i} C_{w}^{z}\right)$. Thus, by saturation for $\mathrm{R} \preccurlyeq$ there exists $z \leqslant k$ and $w \in\left\{1, \ldots, n_{z}\right\}$ such that $B=C_{w}^{z}$ and $A \in \Sigma_{z}$. Let us consider the world $S_{z, w}$ associated to this nested sequent, and the sphere to which $S_{z, w}$ belongs: $\alpha=\left\{S_{k, 1}, \ldots S_{k, n_{k}} \ldots, S_{z, 1}, \ldots, S_{z, n_{z}}\right\}$. By inductive hypothesis, $\mathcal{M}_{N} \Vdash B$, and thus $\alpha \Vdash^{\exists} B$. Moreover, since $\operatorname{set}\left(\Sigma_{z}\right) \subset \operatorname{set}\left(\Sigma_{z+1}\right) \subset \cdots \subset$ $\operatorname{set}\left(\Sigma_{k}\right)$ and by inductive hypothesis, it holds that for all $S_{l, q}$, for $l \in\{z, \ldots, k\}$ and $q \in\left\{1, \ldots, n_{l}\right\}, S_{l, q} \nVdash A$. Since no worlds in $\alpha$ validate $A, \alpha \nVdash^{\exists} A$.

Corollary 1. Let $S=\Gamma \Rightarrow \Delta,\left[G_{1}\right]^{i_{1}}, \ldots,\left[G_{n}\right]^{i_{n}}$ be a saturated nested sequent and $\mathcal{M}_{N}$ a model as defined above. Then, for all $S_{j} \in W$ it holds that $\mathcal{M}_{N}, S_{j} \nVdash$ $\left(S_{j}\right)^{\text {int }}$, and $\mathcal{M}_{N}, S \nVdash(S)^{i n t}$.

Completeness of $\mathcal{N}_{\mathrm{CDL}}$ follows immediately: by Lem. 3, backwards proof search terminates, yielding a derivation or a saturated sequent. In the former case the formula is derivable; in the latter case, we obtain a countermodel using Cor. 1. Moreover, the completeness proof constructs a finite countermodel from a saturated sequent, and thereby also shows the finite model property of the logic.

Theorem 2 (Completeness). Every valid formula is derivable in $\mathcal{N}_{\mathrm{CDL}}$.

Example 3. We construct the countermodel $\mathcal{M}$ for the underivable sequent $\Rightarrow$ $\operatorname{Bel}_{i}(P \rightarrow Q) \rightarrow \operatorname{Bel}_{i}(Q \mid P)$. By backwards applications of $\mathcal{N}_{\mathrm{CDL}}$ rules we obtain the following saturated sequent, where we assume $\preccurlyeq_{i}$ binds stronger than $\wedge$ :

$$
S=P \wedge \neg Q \preccurlyeq i P, \operatorname{Block}_{i}(S) \Rightarrow\left[P \Rightarrow Q, \operatorname{Block}_{i}(S)\right]^{i},\left[\top \Rightarrow P, \operatorname{Block}_{i}(S)\right]^{i}
$$

where $\operatorname{Block}_{i}(S)=\left(\perp \triangleleft_{i} P \wedge \neg Q\right),\left(P \wedge \neg Q, P, \perp \triangleleft_{i} \top\right)$. Let $S_{1}=P \Rightarrow$ $Q, \operatorname{Block}_{i}(S)$ and $S_{2}=\top \Rightarrow P, \operatorname{Block}_{i}(S)$. Then, $W=W_{i}^{S}=\left\{S, S_{1}, S_{2}\right\}$, and $S \sim_{i} S_{1} \sim_{i} S_{2}$. Sequent $S_{1}$ and $S_{2}$ are obtained by jump respectively from the former and latter conditional block in $\operatorname{Block}_{i}(S)$. Since $\{\perp\} \subset\{P \wedge \neg Q, P, \perp\}$ we have that $N_{i}(S)=N_{i}\left(S_{1}\right)=N_{i}\left(S_{2}\right)=\left\{\left\{S_{2}\right\},\left\{S_{2}, S_{1}\right\}, W_{i}^{S}\right\}$. By definition, $P$ is true only at world $S_{2}$, and $Q$ is false at all the worlds. It holds that i) $\mathcal{M}, S \Vdash \operatorname{Bel}_{i}(P \rightarrow Q)$, i.e., that there exists an $\alpha \in N_{i}(S)$ such that $\alpha \Vdash^{\forall} P \rightarrow Q$. Neighbourhood $\left\{S_{2}\right\}$ satisfies the condition. It also holds that ii) $\mathcal{M}, S \nVdash \operatorname{Bel}_{i}(Q \mid P)$, i.e., that there exists an $\alpha \in N_{i}(S)$ such that $\alpha \Vdash^{\exists} P$ and that for all $\beta \in N_{i}(S)$ it holds that $\beta \Vdash^{\exists} P \wedge \neg Q$. The former condition is satisfied by the neighbourhood $\left\{S_{2}, S_{1}\right\}$, and all neighbourhoods satisfy the latter condition. Since i) and ii) hold for all the worlds in the model, $\mathcal{M}$ is a countermodel for the sequent. 


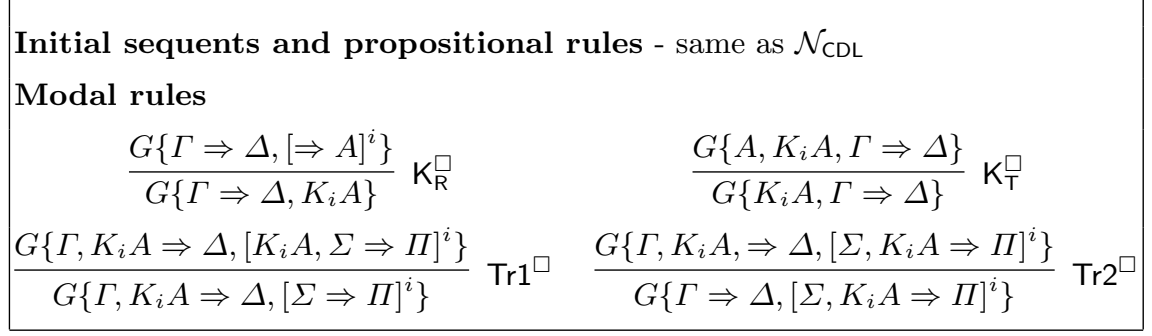

Fig. 3. Rules of $\mathcal{N}_{\mathrm{S} 5_{\mathrm{i}}}$

\section{Relationship with S5 $_{i}$}

As mentioned, the operator $K_{i}$ can be defined by $K_{i} A=\perp \preccurlyeq{ }_{i} \neg A$. If we adopt this definition, restrict the language to $\mathcal{F}_{\mathrm{S}_{i}}=p|\perp| A \rightarrow B \mid K_{i} A$, and apply the rules of $\mathcal{N}_{\mathrm{CDL}}$ to these formulas (Rem. 1), we obtain a nested sequent calculus for a multi-agent modal epistemic logic, where the knowledge operator corresponds to the $\square$ modality. The proof system, called $\mathcal{N}_{\mathrm{S} 5_{\mathrm{i}}}$, captures multiagent logic $\mathrm{S} 5_{i}$.

Nested sequents of $\mathcal{N}_{S_{5} \text { i }}$ are interpreted as $\mathcal{N}_{\mathrm{CDL}}$ nested sequents, with the difference that $\mathcal{N}_{S_{5 i}}$ does not need conditional blocks to capture the simpler semantics of $S 5_{i}$. Observe that the rules of $\mathcal{N}_{S 5_{i}}$ are essentially the multi-agent versions of the standard nested sequent rules for single-agent S5 $[5,16,11]$. But while the nested sequent structure is an overkill for S5, it is necessary to capture $\mathrm{S}_{i}$. To the best of our knowledge, the only published sequent calculus for $\mathrm{S}_{i}$ is Poggiolesi's hypersequent calculus, which uses syntactic labels for the agents [15]. The connection between mono-agent CDL and S5 is known since [10]: As mentioned above, counterfactual logic $\mathbb{V} \mathbb{T} \mathbb{A}$ is the mono-agent system corresponding to CDL. But a Kripke-style accessibility relation $R$ can be obtained from (monoagent) neighbourhood models by setting $R(x, y)$ if and only if $y \in \bigcup N(x)$. For VTA this yields an equivalence relation, thus characterizing modal logic S5. The relation can be used to evaluate formulas $K A$, i.e., formulas $\perp \preccurlyeq \neg A^{7}$. For $A \in \mathcal{F}_{\mathrm{S}_{i}}$, define $T(A) \in \mathcal{F}_{\mathrm{CDL}}$ to be the formula obtained by replacing every occurrence of $K_{i} A$ with $\perp \preccurlyeq_{i} \neg A$. The translation is lifted to nested sequents in the obvious way. By generalizing Lewis' argument to the multi-agent case, we obtain the following:

Lemma 5. If $A$ is a theorem of $\mathrm{S}_{i}$, then $T(A)$ is a theorem of CDL.

Completeness of the nested calculus for $\mathrm{S} 5_{i}$ seems to be unpublished, but considered folklore in the nested sequent community. Using the previous proposition, it can be obtained proof-theoretically from the completeness of $\mathcal{N}_{\mathrm{CDL}}$.

Theorem 3. The calculus $\mathcal{N}_{\mathrm{S5}_{\mathrm{i}}}$ is sound and complete w.r.t. modal logic $\mathrm{S} 5_{i}$.

${ }^{7}$ Evaluating $K A$ at a world $x$ corresponds to evaluating $\perp \preccurlyeq \neg A$ in the outer neighbourhood of $N(x)$. For this reason, Lewis calls S5 the outer modal logic of VTA. 
Proof (Sketch). Soundness can be proved directly (standard). For completeness, we only sketch the main argument. We claim that for a sequent $S=$ $K_{i} A_{1}, \ldots, K_{i} A_{n}, \Gamma \Rightarrow \Delta, K_{i} B_{1}, \ldots, K_{i} B_{m}$, if there is a derivation of $T(S)=$ $\perp \preccurlyeq_{i} \neg A_{1}, \ldots, \perp \preccurlyeq_{i} \neg A_{n}, \Gamma \Rightarrow \Delta, \perp \preccurlyeq_{i} \neg B_{1}, \ldots, \perp \preccurlyeq_{i} \neg B_{m}$ in $\mathcal{N}_{\mathrm{CDL}}$, then there is a derivation of the original sequent $S$ in $\mathcal{N}_{S 5_{i}}$. If $T(S)$ is derivable in $\mathcal{N}_{\mathrm{CDL}}$, then it must have been derived (modulo rule permutations) either by an application of $\mathrm{T}$ or by multiple applications of $\mathrm{R} \preccurlyeq$, followed by applications of $\mathrm{L} \preccurlyeq$ and com, and finally jump. In the former case, the first premiss of the application of $T$ contains a block $\left(\perp \triangleleft_{i} \perp\right)$ and is derivable via jump, while the right premiss modulo propositional rules is just the premiss of $\mathrm{K}_{\mathrm{T}}^{\square}$. In the latter case, after (backwards) applications of $\mathrm{R} \preccurlyeq$, we first reach the sequent:

$$
\perp \preccurlyeq_{i} \neg A_{1}, \ldots, \perp \preccurlyeq_{i} \neg A_{n}, \Gamma \Rightarrow \Delta,\left(\perp \triangleleft_{i} \neg B_{1}\right), \ldots,\left(\perp \triangleleft_{i} \neg B_{m}\right) .
$$

Similarly to the case of $\mathrm{T}$, the left premiss in any (backwards) application of $\mathrm{L} \preccurlyeq$ to a formula $\perp \preccurlyeq{ }_{i} \neg A_{\ell}$ and a block $\left(\perp \triangleleft_{i} \neg B_{k}\right.$ ) is derivable, since it contains the conditional block $\left(\perp \triangleleft_{i} \perp\right)$. The other premiss of an application of $L \preccurlyeq$ is:

$$
\perp \preccurlyeq_{i} \neg A_{1}, \ldots, \perp \preccurlyeq_{i} \neg A_{n}, \Gamma \Rightarrow \Delta,\left(A_{j}, \perp \triangleleft_{i} \neg B_{1}\right), \ldots,\left(\perp \triangleleft_{i} \neg B_{m}\right) .
$$

Exhaustive backwards applications of $\mathrm{L} \preccurlyeq$ yield the sequent

$$
\perp \preccurlyeq{ }_{i} \neg A_{1}, \ldots, \perp \preccurlyeq_{i} \neg A_{n}, \Gamma \Rightarrow \Delta,\left(\Sigma \triangleleft_{i} \neg B_{1}\right), \ldots,\left(\Sigma \triangleleft_{i} B_{m}\right)
$$

where all blocks have the same $\Sigma=\neg A_{1}, \ldots, \neg A_{n}$. Hence the rule of com is not really necessary: with applications of $L \preccurlyeq$ until saturation we obtain the same sequent as with mixed applications of $L \preccurlyeq$ and com. Finally, by applications of jump and of the rules for negation to the above sequent we reach the sequent

$$
T\left(S^{*}\right)=\perp \preccurlyeq{ }_{i} \neg A_{1}, \ldots, \perp \preccurlyeq{ }_{i} \neg A_{n}, \Gamma \Rightarrow \Delta,\left[\Sigma \Rightarrow B_{1}\right]^{i}, \ldots,\left[\Sigma \Rightarrow B_{m}\right]^{i} .
$$

The corresponding $\mathcal{N}_{S 5_{\mathrm{i}}}$ sequent $S^{*}$ is the same sequent that can be obtained from $\Gamma, K_{i} A_{1}, \ldots, K_{i} A_{n} \Rightarrow \Delta, K_{i} B_{1}, \ldots, K_{i} B_{m}$ by applying first rule $\mathrm{K}_{\mathrm{R}}^{\square}$ to all $K_{i} B_{1}, \ldots, K_{i} B_{m}$ and then $\operatorname{Tr} 1^{\square}$ exhaustively on $K_{i} A_{1}, \ldots, K_{i} A_{n}$.

Thus, the nested calculus $\mathcal{N}_{S_{5}}$ simulates by macro-steps $\mathcal{N}_{\mathrm{CDL}}$ derivations in the restricted language $\mathcal{F}_{\mathrm{S}_{i}}$. Since the structure of conditional blocks is not needed, the rules of com, $\mathrm{Tr}_{3}$ and $\mathrm{Tr}_{4}$ become superfluous and have no corresponding rules in $\mathcal{N}_{S 5_{\mathrm{i}}}$. Rule $\operatorname{Tr}^{\square}$ simulates rule $\operatorname{Tr}_{2}$.

\section{Conclusions}

We have presented the first internal calculus $\mathcal{N}_{\mathrm{CDL}}$ for the multi-agent logic of conditional beliefs CDL. The calculus manipulates nested sequents, where the nesting is determined by nested beliefs of different agents. The calculus provides a decision procedure for the logic. Since CDL contains as a fragment multi-agent $\mathrm{S} 5_{i}$, by specialising the rules of $\mathcal{N}_{\mathrm{CDL}}$ to that fragment we obtain a natural internal calculus for $\mathrm{S} 5{ }_{i}$. CDL logic in itself can be extended to formalise the dynamics of beliefs induced by different kinds of announcements [1]. We plan to study how to extend our calculus to deal with the dynamic extension of CDL. 


\section{References}

1. Baltag, A., Smets, S.: Conditional doxastic models: A qualitative approach to dynamic belief revision. Electronic Notes in Theoretical Computer Science 165, 5-21 (2006)

2. Baltag, A., Smets, S.: A qualitative theory of dynamic interactive belief revision. Logic and the foundations of game and decision theory (LOFT 7) 3, 9-58 (2008)

3. Baltag, A., Smets, S., et al.: The logic of conditional doxastic actions. Texts in Logic and Games, Special Issue on New Perspectives on Games and Interaction 4, 9-31 (2008)

4. Board, O.: Dynamic interactive epistemology. Games and Economic Behavior 49(1), 49-80 (2004)

5. Brünnler, K.: Deep sequent systems for modal logic. Arch. Math. Log. 48, 551-577 (2009)

6. Girlando, M., Lellmann, B., Olivetti, N., Pozzato, G.L.: Standard sequent calculi for Lewis' logics of counterfactuals. In: Michael, L., Kakas, A. (eds.) Logics in Artificial Intelligence. JELIA 2016., Lecture Notes in Computer Science, vol. 10021, pp. 272-287. Springer, Cham (2016)

7. Girlando, M., Lellmann, B., Olivetti, N., Pozzato, G.L.: Hypersequent calculi for lewis' conditional logics with uniformity and reflexivity. In: International Conference on Automated Reasoning with Analytic Tableaux and Related Methods. pp. 131-148. Springer (2017)

8. Girlando, M., Negri, S., Olivetti, N., Risch, V.: The logic of conditional beliefs: Neighbourhood semantics and sequent calculus. In: Advances in Modal Logic. pp. 322-341 (2016)

9. Girlando, M., Negri, S., Olivetti, N., Risch, V.: Conditional beliefs: from neighbourhood semantics to sequent calculus. The Review of Symbolic Logic pp. 1-44 (2018)

10. Lewis, D.K.: Counterfactuals. Blackwell (1973)

11. Marin, S., Straßburger, L.: Label-free modular systems for classical and intuitionistic modal logics. In: Goré, R., Kooi, B.P., Kurucz, A. (eds.) AiML 10. pp. 387-406. College (2014)

12. Negri, S.: Proof theory for non-normal modal logics: The neighbourhood formalism and basic results. IFCoLog Journal of Logic and its Applications (2017)

13. Olivetti, N., Pozzato, G.L.: A standard internal calculus for Lewis' counterfactual logics. In: Nivelle, H.D. (ed.) TABLEAUX 2015, Lecture Notes in Artificial Intelligence, vol. 9323, pp. 270-286. Springer International Publishing (2015)

14. Pacuit, E.: Neighbourhood semantics for modal logics. Springer (2017)

15. Poggiolesi, F.: A cut-free simple sequent calculus for modal logic s5. The Review of Symbolic Logic 1(1), 3-15 (2008)

16. Poggiolesi, F.: The method of tree-hypersequents for modal propositional logic. In: Makinson, D., Malinkowski, J., Wansing, H. (eds.) Towards Mathematical Philosophy, Trends In Logic, vol. 28, pp. 31-51. Springer Science + Business Media B.V. (2009)

17. Stalnaker, R.: Belief revision in games: forward and backward induction 1. Mathematical Social Sciences 36(1), 31-56 (1998) 